\title{
Modelo Demanda-Controle e estresse ocupacional entre profissionais de enfermagem: revisão integrativa
}

Demand-Control model and occupational stress among nursing professionals: integrative review Modelo Demanda-Controle y estrés laboral entre profesionales de enfermería: revisión integrativa

\section{Denise Rodrigues Costa Schmidt'}

' Universidade Estadual de Londrina, Hospital Universitário. Londrina-PR, Brasil.

Submissão:13-06-2013Aprovação:27-08-2013

\section{RESUMO}

O modelo Demanda-Controle, com grande poder explanatório, tem como objetivo a avaliação do estresse ocupacional. Buscou-se conhecer, através de uma revisão integrativa, a produção científica sobre a utilização do modelo Demanda-Controle para investigação do estresse ocupacional entre profissionais de enfermagem, no período de 2000 a 2011. Dos 16 estudos selecionados, a maioria (cinco artigos) foi publicada em 2009. A Job Stress Scale e o Job Content Questionnaire foram utilizados em nove e sete estudos, respectivamente. Houve uma grande diversidade na forma de análise dos dados. As dimensões Demanda e Controle e sua associação com problemas relacionados à saúde do trabalhador foram avaliadas em $56,25 \%$ dos estudos, dos quais 37,5\% estavam relacionados à saúde mental. Os artigos apontam a necessidade de produção científica nacional, sugerindo a atuação dos pesquisadores em estudos de intervenção que possibilitem a redução do estresse laboral e, consequentemente, melhoria do estado de saúde mental dos trabalhadores.

Descritores: Estresse; Enfermagem; Saúde Ocupacional.

\section{ABSTRACT}

The Demand Control model aims to evaluate the occupational stress. This study aimed to know, through an integrative review of the literature, the scientific production about the Demand Control Model to investigation occupational stress among nursing professionals from 2000 to 2011.Of the 16 selected studies, five were published in 2009. Of these studies, 56.25\% assessed the Demand and Control dimensions and their correlations with workers' health problems; $37.5 \%$ of these studies were related with mental health. The results showed a lack of national publications. We recommend that authors conduct experimental studies to reduce the occupational stress for better conditions of workers' mental health.

Key words: Stress; Nursing; Occupational Health.

\section{RESUMEN}

El modelo Demanda-Controle tiene como objetivo la evaluación del estrés laboral. En este estudio se procuró conocer, a través de una revisión integrativa, la producción científica sobre lo estrés laboral de los profesionales de enfermería, en el período de 2000 a 2011. De los 16 estudios seleccionados, cinco artículos fueron publicados en 2009. Entre los estudios, 56,25\% evaluaron las dimensiones Demanda y Controle y su asociación con problemas relacionados con la salud de los trabajadores; $37,5 \%$ de estos estudios estaban relacionados con la salud mental. Los artículos apuntan a la necesidad de producción científica, lo que sugiere estudios de intervención que permitan la reducción de estrés laboral y, en consecuencia, la promoción de apoyo para la mejora del estado de salud mental de los trabajadores.

Palabras clave: Estrés; Enfermería; Salud Ocupacional. 


\section{INTRODUÇÃO}

O estresse ocupacional tem afetado cada vez mais a saúde do trabalhador e a economia de países desenvolvidos e em desenvolvimento. Inúmeras pesquisas têm sido desenvolvidas buscando compreender o impacto do estresse nas organizações e no adoecimento do trabalhador.

Entende-se que o estresse ocupacional é aquele oriundo do ambiente de trabalho, ou seja, é um conjunto de fenômenos que se apresentam no organismo do trabalhador e que, por este motivo, pode afetar sua saúde. Os principais fatores geradores de estresse presentes no ambiente de trabalho envolvem os aspectos da organização, administração e sistema de trabalho e da qualidade das relações humanas, porém a quantidade de estresse que cada pessoa experimenta pode ser modulada por fatores como sua experiência no trabalho, o nível de habilidade, o padrão de personalidade e a autoestima ${ }^{(1)}$.

A avaliação desses aspectos e de sua influência na saúde dos trabalhadores tem avançado consideravelmente, porém sua mensuração ainda configura-se como um desafio para a epidemiologia social. Tal mensuração é frequentemente embasada por construtos teóricos, que geram modelos testados em diversos campos empíricos. Um dos modelos com maior poder explanatório e amplamente utilizado na literatura internacional como medida de condições psicossociais do ambiente do trabalho é o modelo Demanda-Controle (D-C). Karasek, no inicio da década de 1970, considerando o enfoque limitado dos modelos unidimensionais existentes, propôs esse modelo bidimensional baseado na abordagem simultânea do controle sobre o trabalho e das demandas psicológicas ${ }^{(2)}$.

Posteriormente, o modelo passou a incluir uma terceira dimensão: a percepção de apoio social do trabalho. Segundo os autores $^{(2)}$, a integração social, a confiança no grupo, a ajuda na realização das tarefas por parte de colegas e superiores poderiam atuar como protetoras (moderadoras) dos efeitos do desgaste no trabalho sobre a saúde.

Conforme concebidas no modelo, as duas principais dimensões, Demanda e Controle, abrangem aspectos específicos do processo de trabalho. O controle pode ser definido como a amplitude de decisão que o trabalhador possui em relação a dois aspectos: o uso de habilidades e a autoridade decisória ou autonomia para tomada de decisões sobre seu próprio trabalho. $\mathrm{O}$ uso de habilidades é o grau pelo qual o trabalho envolve aprendizagem de coisas novas, repetitividade, criatividade, tarefas variadas e o desenvolvimento de habilidades especiais individuais; a autoridade decisória abarca a habilidade individual para a tomada de decisão sobre o próprio trabalho, a influência do grupo de trabalho e a influência na política gerencial ${ }^{(2)}$.

A demanda psicológica se refere às exigências psicológicas que o trabalhador enfrenta na realização das suas tarefas, envolvendo pressão de tempo, nível de concentração requerida, interrupção das tarefas e necessidade de se esperar pelas atividades realizadas por outros trabalhadores ${ }^{(2)}$.

No modelo de Karasek existem quatro tipos básicos de experiências no trabalho gerados pela combinação de alto e baixo nível de demanda psicológica e de controle, quais sejam: trabalho de alta exigência; trabalho ativo; trabalho passivo e trabalho com baixa exigência. Os trabalhadores que se defrontam com alta demanda psicológica, combinada com um baixo controle sobre o trabalho correm maior risco de apresentar problemas de saúde física e mental decorrentes do estresse (alto desgaste ou trabalho de alta exigência). Os trabalhos considerados ativos são aqueles que possuem altas demandas psicológicas, mas que permitem ao trabalhador ter uma ampla possibilidade de decisão sobre como e quando desenvolver suas tarefas, bem como quando usar todo seu potencial intelectual para isso. Por outro lado, os trabalhos que combinam baixa demanda e baixo controle, chamados de trabalho passivo, são vistos como indutores de declínio na atividade geral do individuo, pois o trabalhador sente-se num estado de apatia, seja pela ausência de desafios significantes e de permissão para atuações com energia, seja pela rejeição sistemática às suas iniciativas de trabalho. Por fim, os trabaIhos considerados como sendo de baixo desgaste são aqueles que combinam poucas demandas psicológicas e muito controle sobre o trabalho ${ }^{(2)}$.

Difundido internacionalmente entre pesquisadores de diversas áreas, o modelo D-C já foi utilizado entre indivíduos da população geral e entre trabalhadores de diversos setores ${ }^{(3-8)}$, e também entre profissionais da área saúde. Na enfermagem, o estresse ocupacional, avaliado a partir do modelo Demanda-Controle, tem sido associado a doenças musculoesqueléticas, burnout, ansiedade, depressão e senso de coerência ${ }^{(9-13)}$.

Dois diferentes instrumentos traduzidos e adaptados para o português brasileiro são utilizados no Brasil para mensurar o estresse ocupacional segundo o modelo D-C: o Job Content Questionnaire $(\mathrm{JCQ})^{(3)}$, que pode ser acessado pelo site $W w W$. jcqcenter.org e a sua versão reduzida denominada Job Stress Scale (JSS) ${ }^{(14)}$. O primeiro possui 49 questões: 17 avaliam controle; nove avaliam demandas psicológicas, cinco demandas físicas; 11 avaliam suporte social; seis indagam sobre insegurança no trabalho e uma questão investiga o nível de qualificação requerido para a atividade de trabalho realizada. A JSS foi elaborada originalmente na Suécia por Thores Theörell, em 1988, e trata-se de uma versão resumida do JCQ, contendo 17 itens: cinco para avaliar demanda; seis para avaliação da dimensão controle e seis para a avaliação da dimensão suporte social ${ }^{(14)}$.

O fato de existirem diferentes formas de avaliar as categorias de exposição ao estresse ocupacional torna o modelo Demanda-Controle demasiadamente complexo, exigindo estudos que auxiliem sua utilização.

Diante do exposto e ciente da importância desse modelo para a avaliação do estresse laboral e da necessidade de divulgação do mesmo entre enfermeiros, elaborou-se este estudo com o objetivo de avaliar a utilização do modelo Demanda-Controle para investigação do estresse ocupacional na enfermagem brasileira.

\section{MÉTODO}

Estudo de revisão integrativa, a qual foi escolhida porque corresponde a um método de pesquisa que viabiliza análise de pesquisas científicas de modo sistemático e amplo, 
favorecendo a caracterização do conhecimento produzido sobre a utilização do Modelo Demanda - Controle para avaliação do estresse ocupacional. Permitindo ainda revelar lacunas do conhecimento sobre a temática estudada ${ }^{(15)}$.

Para a realização deste estudo, seis etapas foram percorridas: estabelecimento do problema de revisão; seleção da amostra; categorização dos estudos; análise dos resultados; apresentação e discussão dos resultados; e por fim, apresentação da revisão(16).

O estudo foi direcionado pelo seguinte questionamento: "Qual o conhecimento científico produzido, no período de 2000 a 2011, sobre o estresse ocupacional na enfermagem brasileira com a utilização do modelo Demanda-Controle?"

No desenvolvimento desse estudo selecionamos as pesquisas divulgadas por meio de resumos publicados em anais de eventos científicos, artigos, teses e dissertações, publicados na íntegra ou em suas versões resumidas e indexados nas seguintes bases de dados: Literatura Latino Americana em Ciências da Saúde (LILACS), Medical Literature Analysis and Retrieval System on-line (MEDLINE), Scientific Eletronic Library Online (SciELO) e banco de teses da CAPES.

Para seleção do material científico encontrado foram utilizados os seguintes critérios de inclusão: artigos científicos nacionais, publicados em português e inglês, durante o período de 2000 a fevereiro de 2011, que avaliaram o estresse ocupacional por meio das versões validadas e adaptadas para a língua portuguesa do JCQ e da JSS; com resumos ou textos na íntegra disponíveis nas bases de dados selecionadas. Foram empregadas as seguintes palavras-chave para a busca dos estudos: estresse ocupacional; estresse; enfermagem e Karasek.

Para alcance do objetivo proposto elaborou-se um instrumento, o qual foi submetido a validação aparente e de conteúdo por três juízes enfermeiros, contendo dados relacionados à identificação da pesquisa, objetivo e características metodológicas, instrumento utilizado, população avaliada, análise dos dados e principais resultados. A apresentação dos resultados e discussão dos dados obtidos foi realizada de forma descritiva e em quadro sinóptico.

\section{RESULTADOS}

No período delimitado, foram identificados 200 estudos que apresentavam como tema principal o estresse ocupacional de acordo com as bases teóricas do modelo Demanda-Controle. Destes estudos, excluímos 167 por não terem sido realizados no Brasil. Foram excluídos outros 17 estudos: um por se tratar de revisão de literatura sobre o tema; outro por abordar o processo de adaptação cultural da JSS, não atendendo aos objetivos desse estudo; dez por se tratar da avaliação do estresse entre outros profissionais; e cinco por terem sido encontrados em mais de uma base de dados. Foram avaliados, portanto, 16 estudos.

Das 16 pesquisas selecionadas, 12 eram artigos científicos, todos localizados na íntegra; três eram dissertações de mestrado, das quais uma foi localizada na íntegra; e uma era tese de doutorado, localizada em sua versão resumida.

O Quadro 1 apresenta os estudos analisados, segundo os autores, ano de publicação, instrumentos utilizados, população estudada, análise dos dados e os principais resultados encontrados.

Observou-se uma lacuna de publicações referente ao estresse ocupacional com a utilização do modelo Demanda-Controle na enfermagem nos anos de 2000 a 2002, e 2004 a 2005. A frequência de publicações ficou distribuída da seguinte forma: 2003 (1 artigo); 2006 (1 artigo); 2007 (2 artigos); 2008 (3 artigos), 2009 (5 artigos), 2010 (1 artigo) e 2011 (3 artigos), evidenciando que as publicações sobre o tema tomaram maior impulso a partir de 2007, atingindo maior número em 2009 (31,25\%). Embora se observe ainda a necessidade de divulgação do modelo Demanda-Controle na enfermagem, haja vista que o número total de publicações ainda é pequeno quando comparado aos estudos internacionais, observa-se que o ano de 2011 poderá mudar este cenário, contribuindo ainda mais para a divulgação do tema.

Os instrumentos de medida foram utilizados de forma equilibrada. O Job Content Questionnaire foi utilizado em sete estudos $^{(10,17,19,24,26-27,31)}$ e a Job Stress Scale foi utilizada em nove estudos $^{(18,20-23,25,28-30)}$. Quanto às unidades de trabalho avaliadas, constatou-se que a maioria dos estudos avaliou os trabaIhadores de unidades gerais, abertas $(68,75 \%)$; sendo que as unidades de centro cirúrgico ${ }^{(25,27)}$ e terapia intensiva ${ }^{(21,26)}$ foram abordadas isoladamente em dois estudos e junto com outras unidades em um estudo(18).

De acordo com a avaliação dos estudos, constatou-se que a principal forma de avaliar os aspectos psicossociais relacionados ao trabalho através da utilização do modelo Demanda-Controle nos estudos que utilizaram o Job ContentQuestionnaire foi por meio da categorização das dimensões Demanda e Controle, a partir das quais foram identificados os trabalhadores ativos, passivos, de alto desgaste e baixo desgaste.

Com relação à Job Stress Scale, observou-se uma maior variação na forma de análise, ou seja, um estudo ${ }^{(22)}$ utilizou o escore contínuo das dimensões Demanda e Controle; cin$\mathrm{CO}^{(18,20-21,23)}$ realizaram a categorização das dimensões, a partir da qual se formaram os quadrantes propostos no modelo Demanda-Controle; outro estudo ${ }^{(25)}$ categorizou as dimensões Demanda e Controle em alta e baixa demanda e alto e baixo controle, utilizando a mediana como ponto de corte, e a seguir classificou os trabalhadores nos três grupos de exposição ao estresse ocupacional, conforme proposto em estudo prévio $^{(19)}$; e, por último, um estudo ${ }^{(29)}$ utilizou a razão entre Demanda e Controle além dos escores das dimensões.

Embora os estudos analisados tenham apresentado a associação de Demanda e Controle com distúrbios osteomusculares, problemas relacionados à saúde mental, acidente com material biológico, capacidade para o trabalho e qualidade de vida, a maioria dos estudos ${ }^{(17-20,24,30)}(37,5 \%)$ avaliou a associação dessas variáveis com os distúrbios psíquicos, constatando que, no geral, a prevalência destes transtornos é mais elevada entre os profissionais com trabalho de alta exigência. Os resultados dos estudos identificaram também que os Distúrbios Psíquicos Menores (DPM) apresentaram associação positiva com Demanda psicológica, ou seja, quanto maior a Demanda, por exemplo, maior a presença de DPM. Nesses estudos observou-se que a prevalência de Transtornos Mentais Comuns (TMC) ficou entre 23,6\% e 26,3\%, 


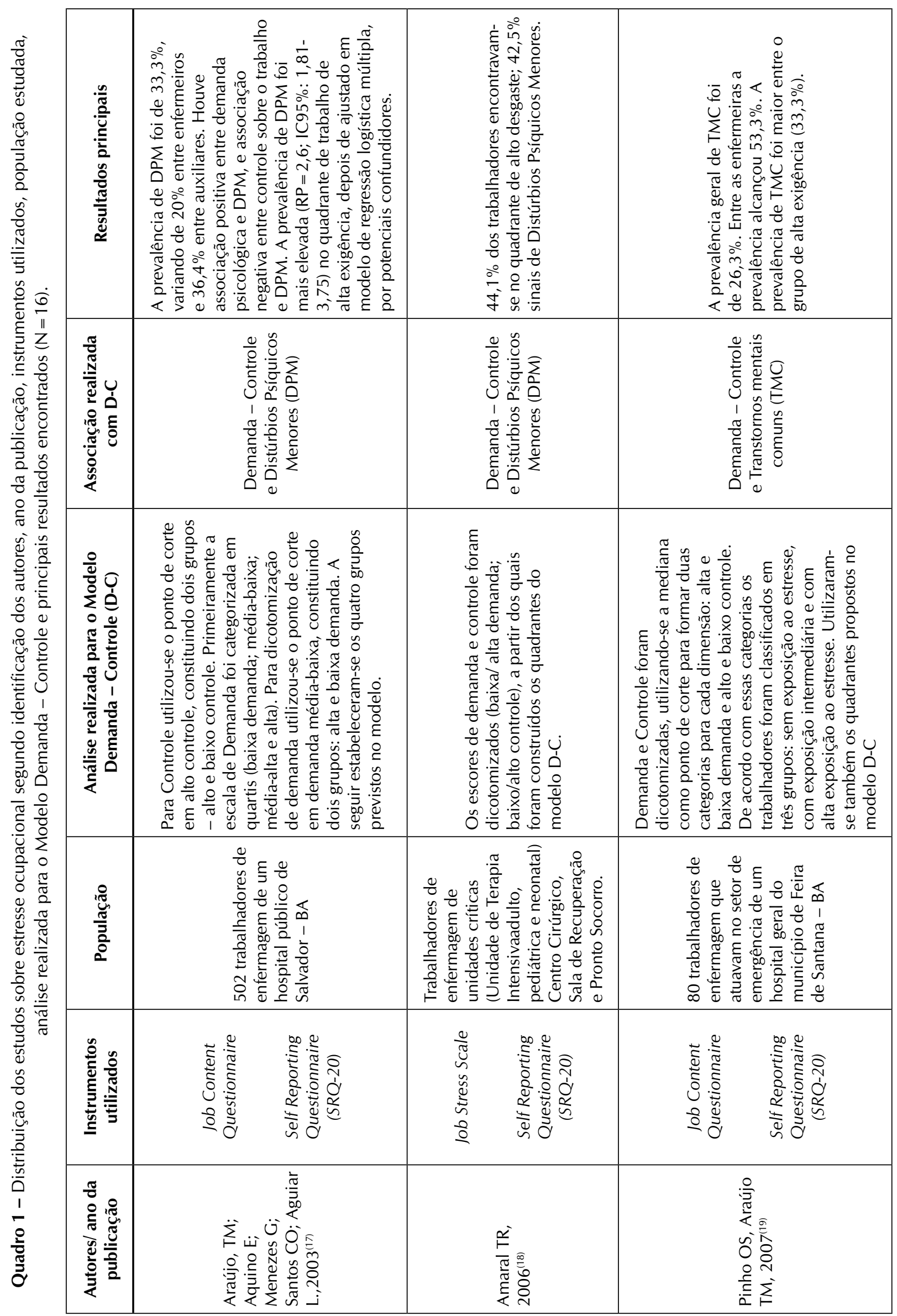




\begin{tabular}{|c|c|c|c|c|c|}
\hline 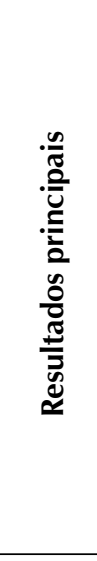 & 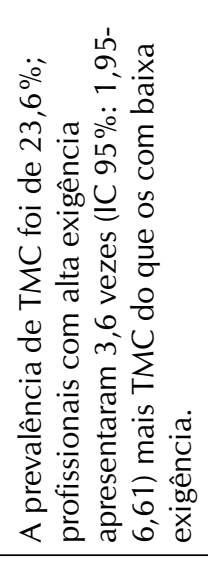 & 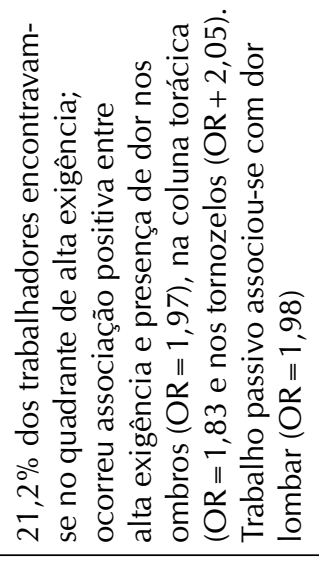 & 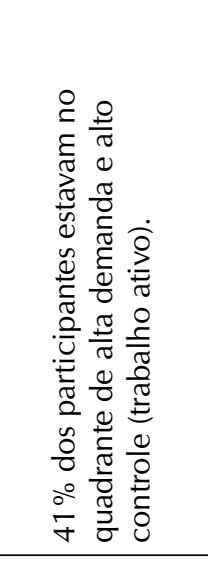 & 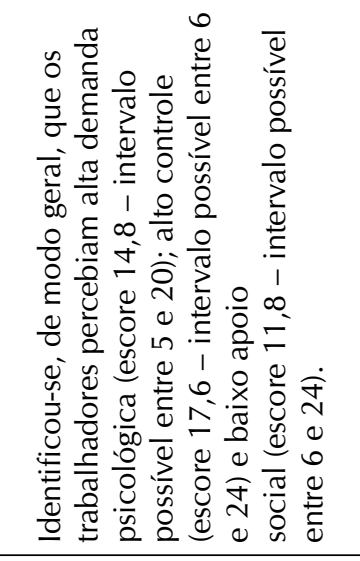 & 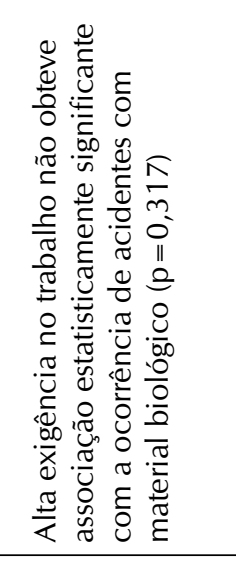 \\
\hline 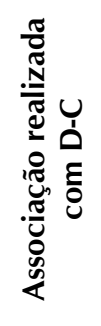 & 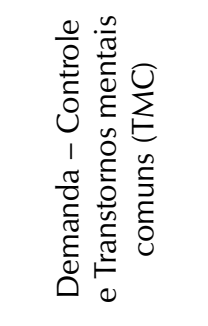 & 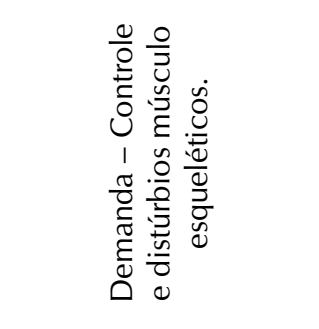 & 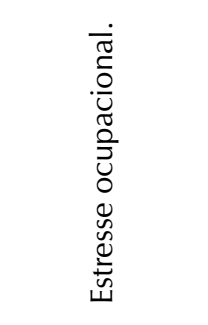 & 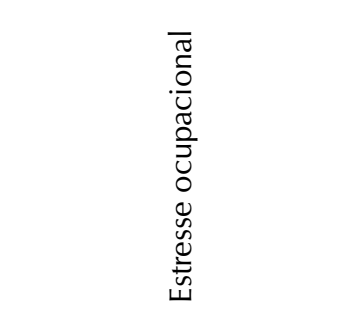 & 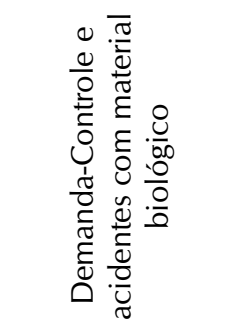 \\
\hline 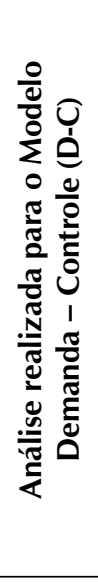 & 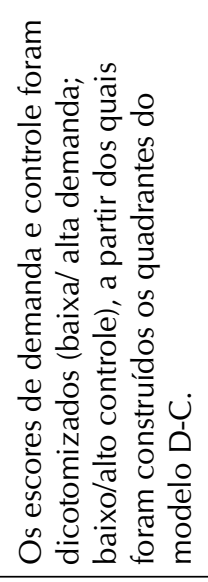 & 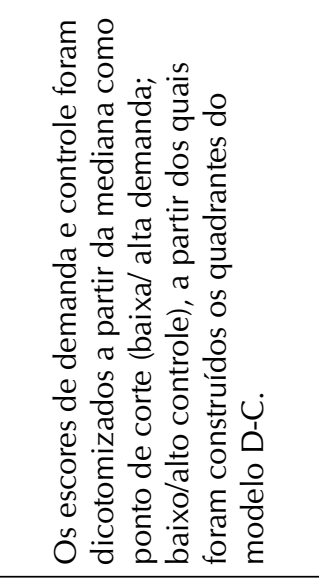 & 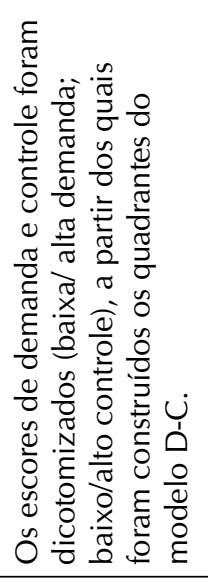 & 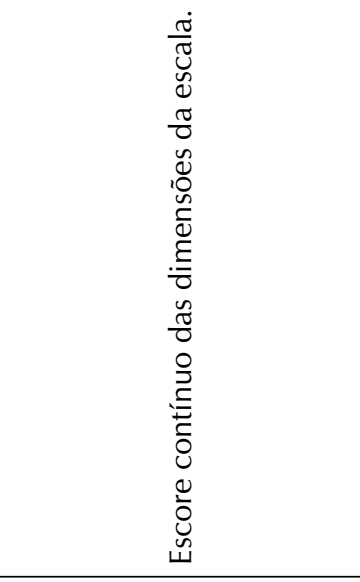 & 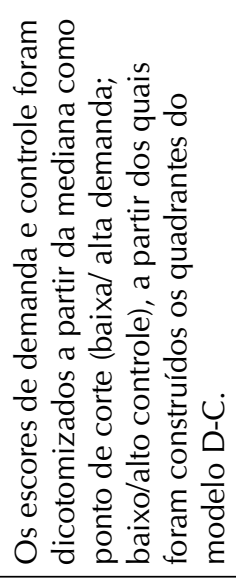 \\
\hline $\begin{array}{l}\text { 웛 } \\
\frac{\pi}{\overline{2}} \\
\frac{0}{0}\end{array}$ & 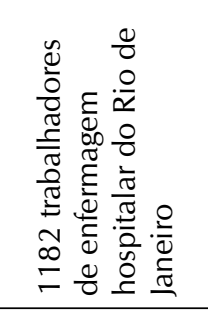 & 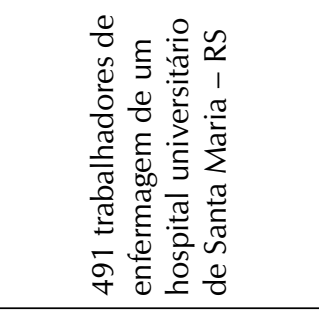 & 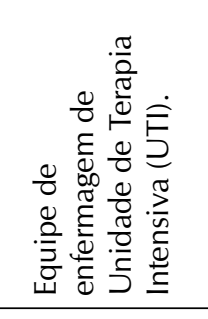 & 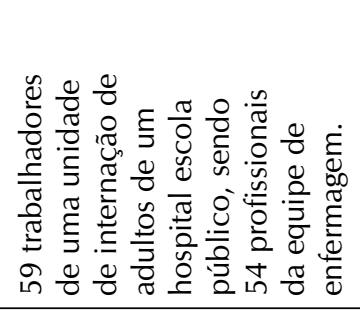 & 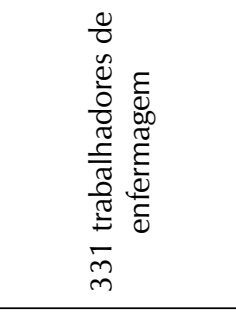 \\
\hline 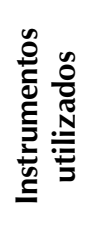 & 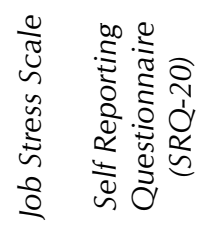 & 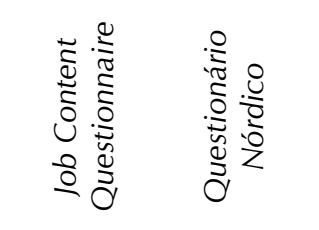 & 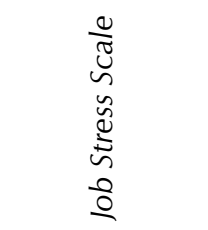 & 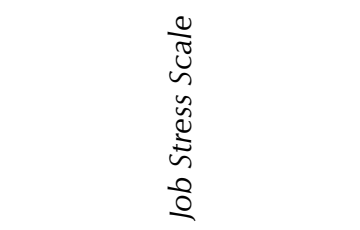 & 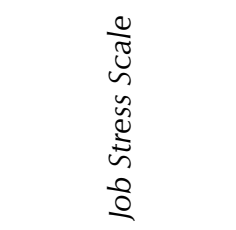 \\
\hline 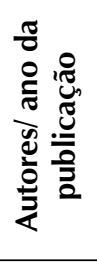 & 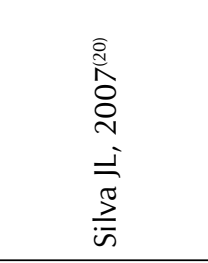 & 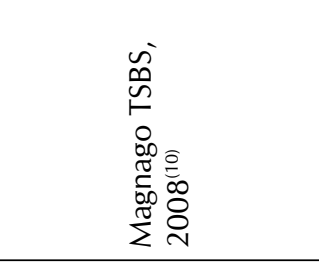 & 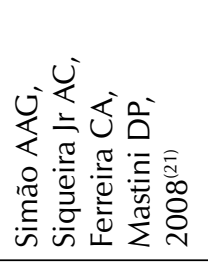 & 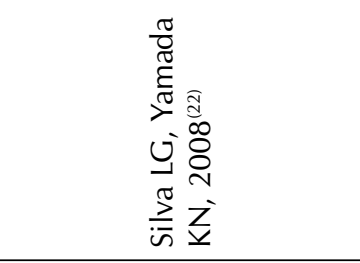 & 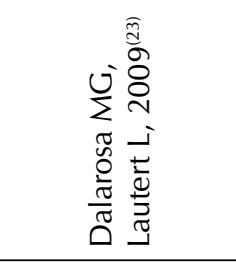 \\
\hline
\end{tabular}




\begin{tabular}{|c|c|c|c|c|}
\hline 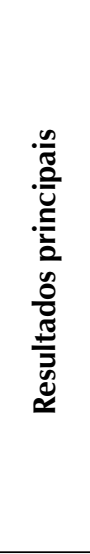 & 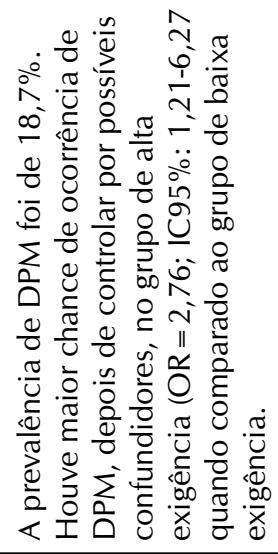 & 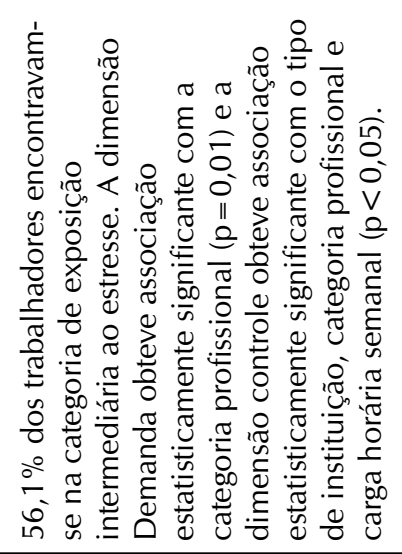 & 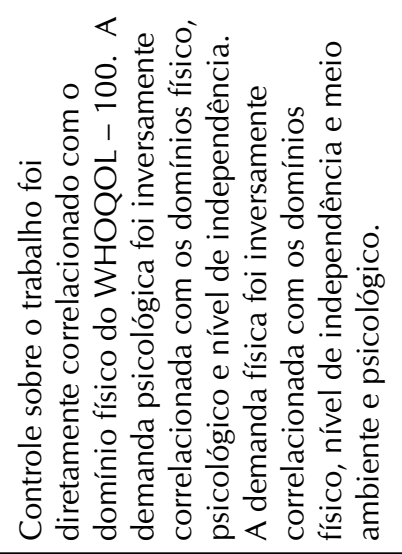 & 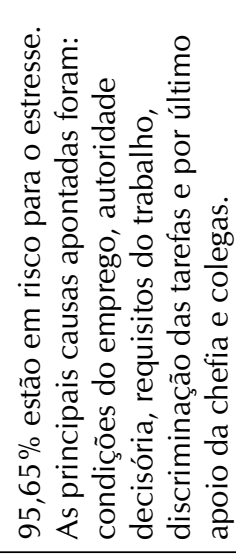 \\
\hline 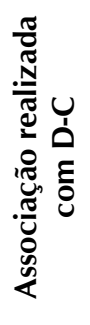 & 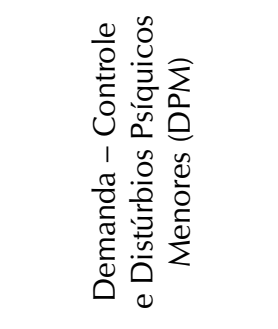 & 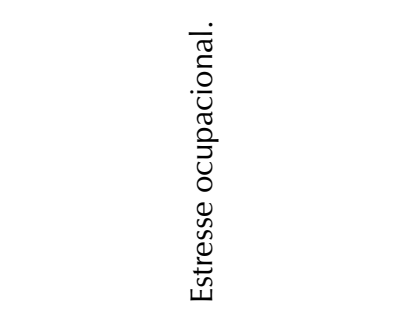 & 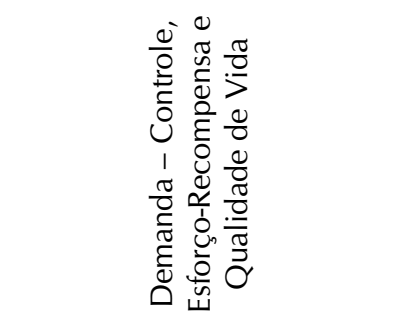 & 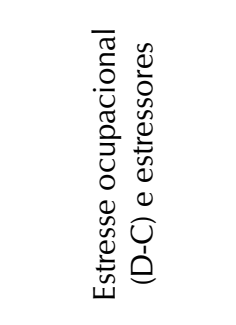 \\
\hline 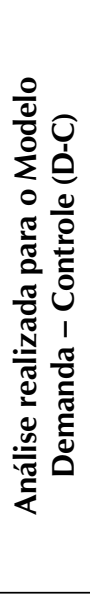 & 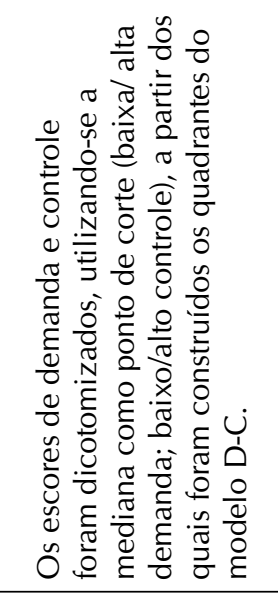 & 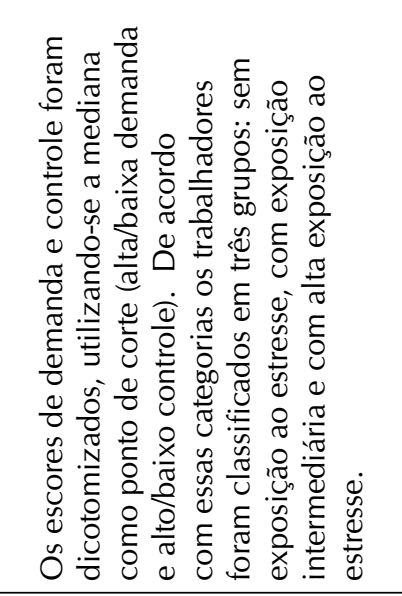 & 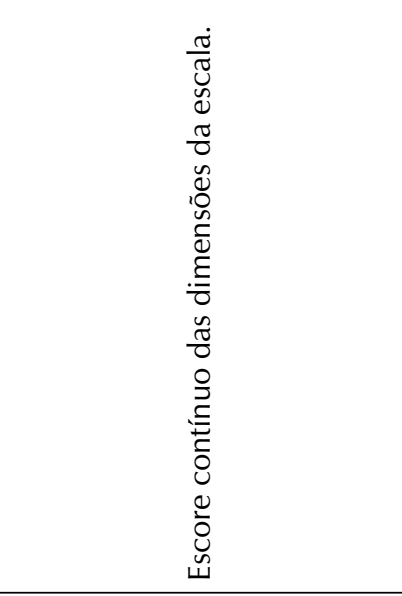 & 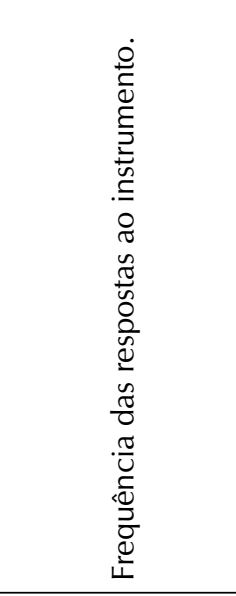 \\
\hline 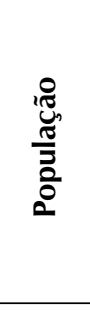 & 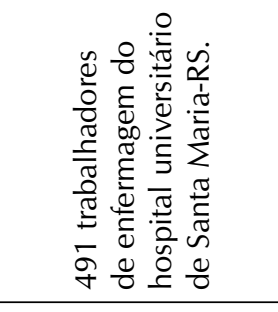 & 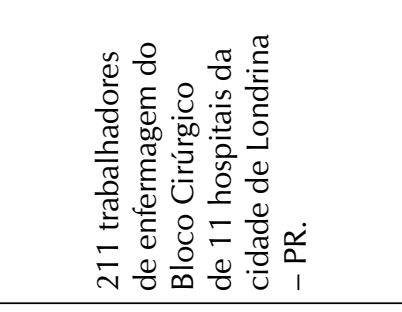 & 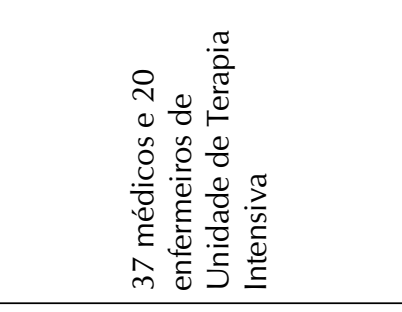 & 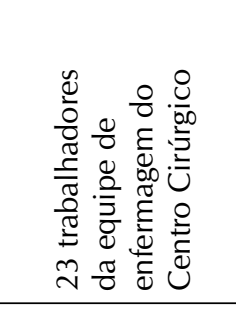 \\
\hline 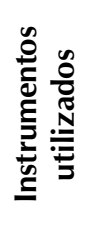 & 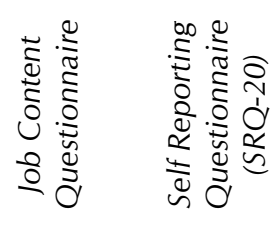 & 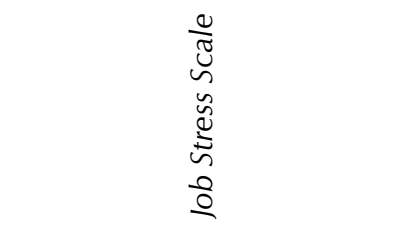 & 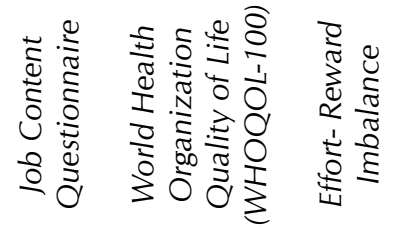 & 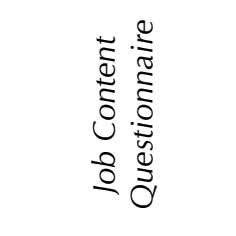 \\
\hline 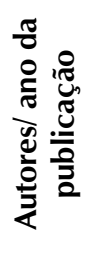 & 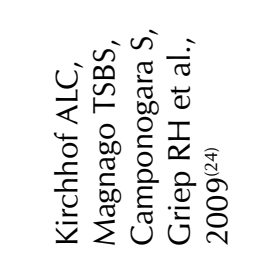 & 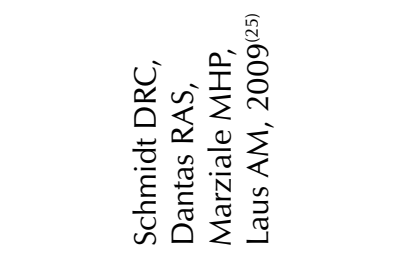 & 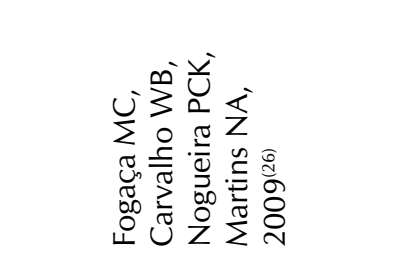 & 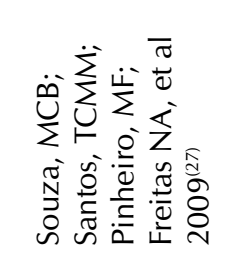 \\
\hline
\end{tabular}




\begin{tabular}{|c|c|c|c|c|}
\hline 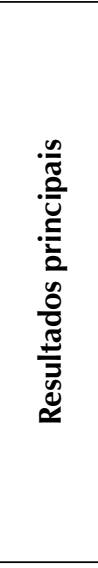 & 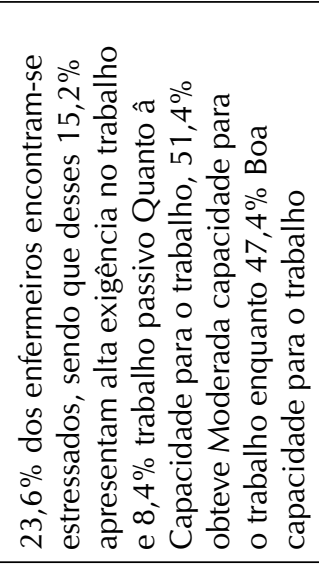 & 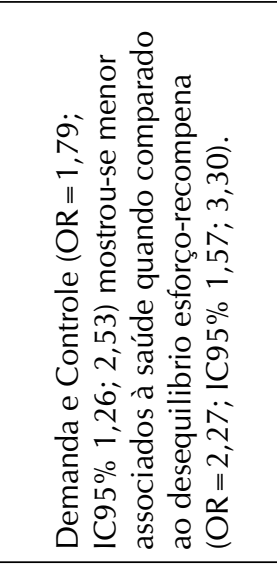 & 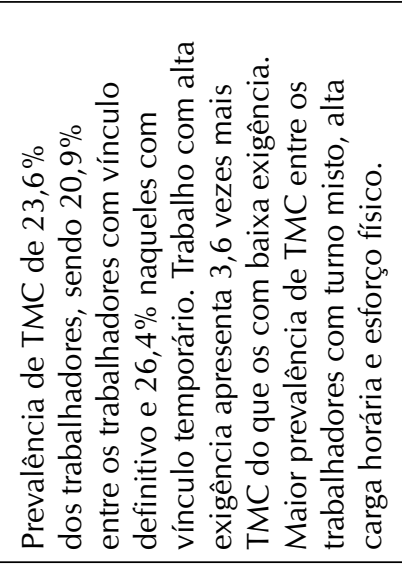 & 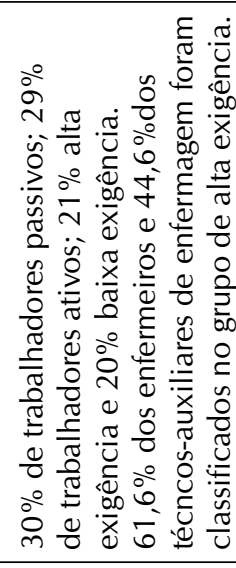 \\
\hline 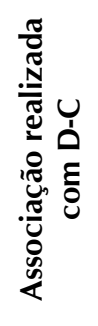 & 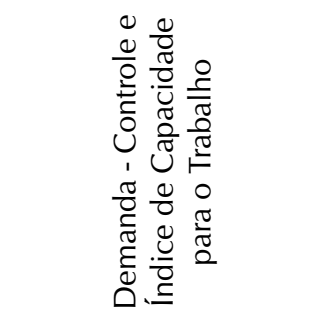 & 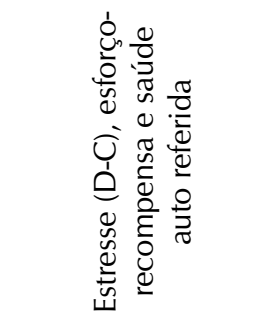 & 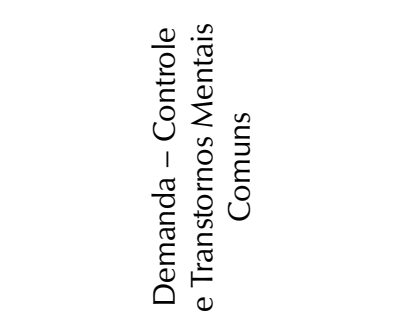 & 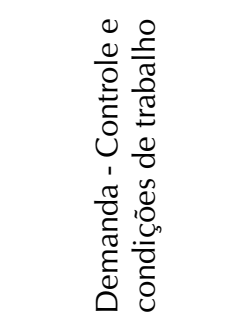 \\
\hline 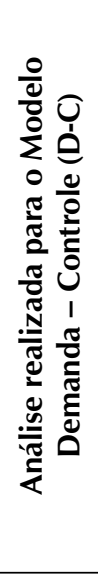 & 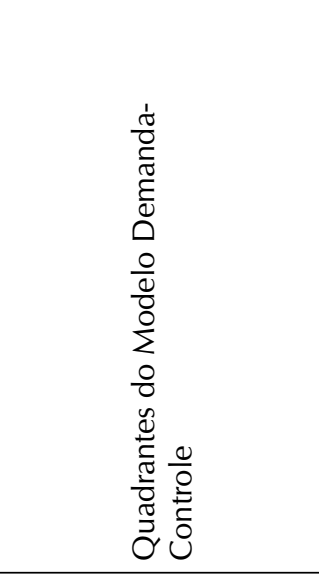 & 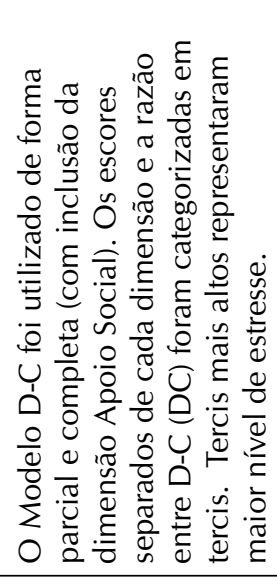 & 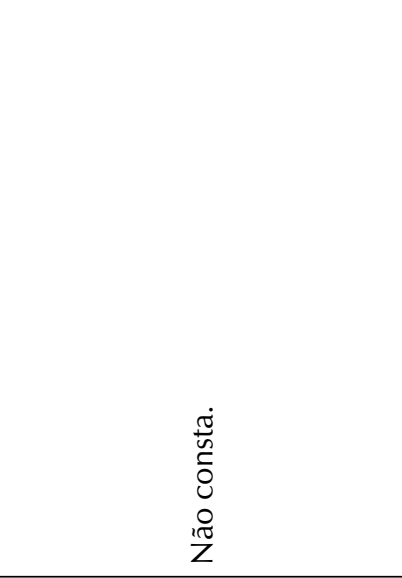 & 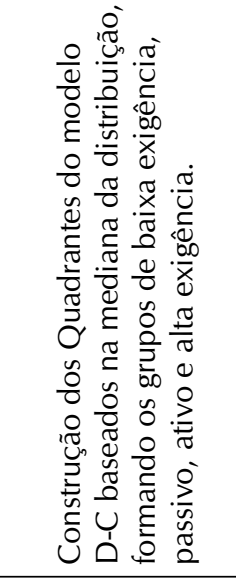 \\
\hline 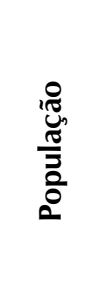 & 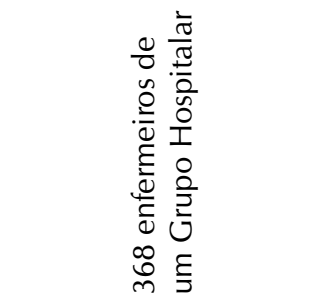 & 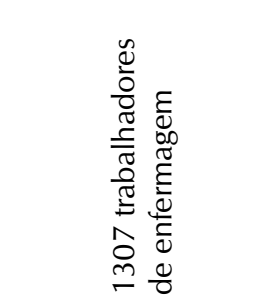 & 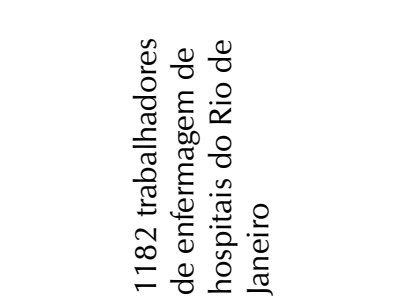 & 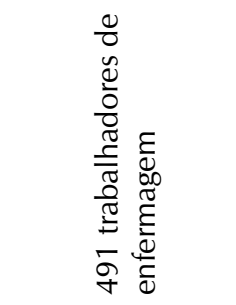 \\
\hline 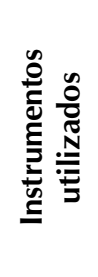 & 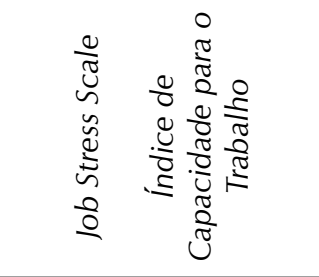 & 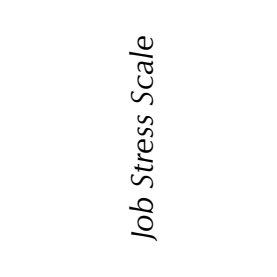 & 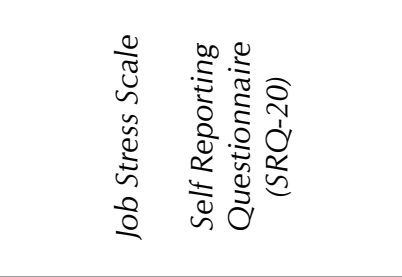 & 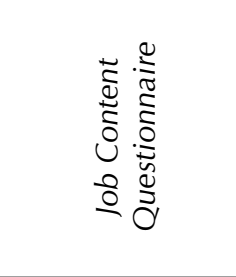 \\
\hline 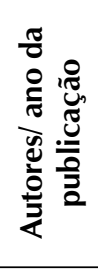 & 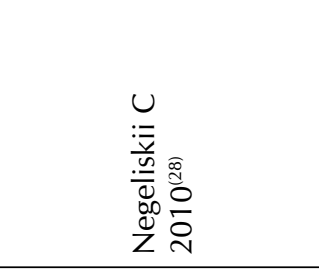 & 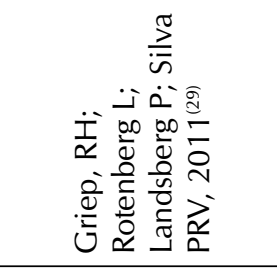 & 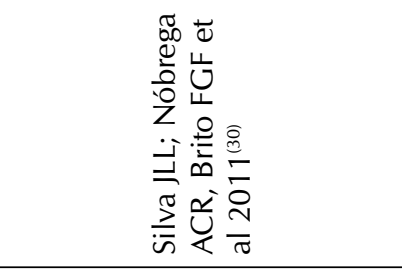 & 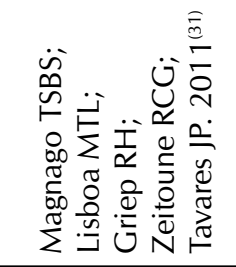 \\
\hline
\end{tabular}


enquanto a prevalência de DPM foi de 18,7\%. Evidenciou-se também que os profissionais de enfermagem encontravam-se no quadrante de alta exigência (21,2\% a 44,1\% dos trabalhadores) e no quadrante de trabalho ativo ( $41 \%$ dos trabalhadores).

Para finalizar a análise dos estudos, subdividiu-se o tema abordado em três categorias: 1- avaliação de Demanda, Controle e aspectos relacionados à saúde do trabalhador (9 artigos - 56,25\% (10,17-20,24,26,29-30); 2- avaliação de Demanda, Controle e aspectos relacionados ao ambiente de trabalho (3 artigos - 18,75\%)(23,28,31); avaliação da presença do estresse ocupacional segundo o modelo D-C (4 artigos - 25\%) $)^{(21-22,25,27)}$.

\section{DISCUSSÃO}

O modelo Demanda-Controle alcançou uma disseminação internacional e tem sido amplamente usado em países como Suécia, Dinamarca, Suíça, Inglaterra, Estados Unidos e Japão, sendo que a expansão do uso do JCQ promoveu a criação de um centro agregador de pesquisadores e de informações sobre o Modelo Demanda-Controle ${ }^{(3)}$.

De acordo com o resultado obtido neste estudo, podemos observar que, no Brasil, os estudos voltados à avaliação dos aspectos psicossociais relacionados ao trabalho por meio da utilização do Modelo Demanda-Controle vêm crescendo gradativamente, principalmente a partir de 2008, tornando-se um modelo investigativo de grande repercussão na área do estresse ocupacional. Apesar desse aspecto, considera-se que estudos desta natureza devem ser realizados com maior frequência para ampliar ainda mais a aplicação do modelo Demanda- Controle e consequentemente melhorar as condições de trabalho dos profissionais investigados.

Com relação a esse aspecto, observamos que o ano de 2011 obteve um número significativo de publicações (três estudos), considerando que foram analisados apenas os dois primeiros meses deste ano. Não podemos, no entanto, deixar de mencionar que o ano de 2010 contribuiu minimamente com publicações nesta área (apenas um estudo), o que poderia comprometer o avanço dessa temática dentro da enfermagem.

O estudo sobre o estresse ocupacional entre trabalhadores de enfermagem se justifica uma vez que esses profissionais, em sua atividade laboral no contexto hospitalar, enfrentam uma exigência emocional adicional devido a uma série de estressores, tais como, problemas de relacionamento e interação com membros de outras equipes, mudanças tecnológicas, falta de recursos humanos e de material, a assistência de enfermagem que envolve a dor, a morte e situações de urgência, interferência na vida pessoal, entre outros ${ }^{(19)}$.

Com relação à análise dos dados dos estudos que fizeram parte desta revisão, verificamos que alguns autores estudaram as dimensões Demanda e Controle como variáveis contínuas, outros utilizaram os quadrantes, a razão D-C e, ainda, a frequência de respostas aos itens do instrumento. No entanto, os motivos pelos quais os autores optaram por uma ou outra forma de analisar a exposição do trabalhador não ficaram bem esclarecidos nos estudos avaliados.

Essa situação foi bastante similar à encontrada internacionalmente, ou seja, há estudos cuja análise dos dados foi realizada por meio da razão entre demanda e controle ${ }^{(32)}$, através da categorização da escala segundo os quadrantes ${ }^{(33)}$ e ainda através dos escores contínuos de demanda e controle ${ }^{(34)}$.

Alguns autores ${ }^{(3)}$ já haviam apontado que a diversidade metodológica, teórica e conceitual predomina nos estudos realizados, ou seja, cada estudo avalia um aspecto específico, ou um mesmo aspecto é avaliado de modo diferente, ou ainda, é dada denominações diversas para um mesmo aspecto. Esses autores assinalaram que tal situação tem dificultado avanços importantes, especialmente aqueles referentes às intervenções no ambiente de trabalho, uma vez que impossibilita a comparação entre os estudos realizados. Considera-se também que a utilização de conceitos e instrumentos distintos para o mesmo constructo dificulta a comparação dos resultados dos estudos e, em consequência, a implementação de medidas de melhoria nas condições de trabalho.

Ainda com relação à análise dos dados aplicada aos estudos encontrados, apenas um estudo utilizou a razão entre Demanda e Controle (Razão D/C). Esse aspecto pode ser justificado uma vez que o uso da razão para análise da exposição do trabalhador implica em definir apenas os dois grupos extremos: o de menor e o de maior desgaste ${ }^{(4)}$. Ao se utilizar essa forma de avaliação os grupos passivos e ativos não são delimitados o que poderia prejudicar a investigação.

Já a escolha pelo uso dos quadrantes é de extrema importância, uma vez que todos os grupos podem ser avaliados. A não inclusão do grupo de trabalhadores passivos que também apresenta baixo controle, além do grupo com maior desgaste, pode dificultar a análise das condições de trabalho e a produção de soluções para os problemas enfrentados ${ }^{(4)}$.

Quanto aos instrumentos de medida, observou-se que o JCQ e a JSS foram utilizadas de forma equilibrada, constatando que apesar do JCQ apresentar um formato longo, com 49 itens, sua utilização não ficou limitada. A existência de versões resumidas para diversos instrumentos internacionalmente conhecidos é uma realidade que amplia a utilização dos mesmos, uma vez que a praticidade e a economia de tempo são fatores importantes, tanto para quem aplica quanto para quem responde o instrumento. No entanto, as versões reduzidas além de manter a representação do construto de interesse, devem preservar adequadas propriedades métricas, o que se observou na versão resumida do $\mathrm{JCQ}^{(14)}$.

O estudo da interação dos problemas relacionados à saúde mental e do comportamento humano no trabalho tem obtido cada vez mais visibilidade nacionalmente, justificando a predominância de pesquisas que abordaram esse aspecto dentre os estudos avaliados.

\section{CONCLUSÃO}

Conclui-se que, apesar dos resultados apontarem para um crescimento do tema estresse ocupacional relacionado ao modelo Demanda-Controle, observou-se uma carência de produção científica nacional, sugerindo-se a necessidade de maior atuação dos pesquisadores em estudos de intervenção que possibilitem a redução do estresse laboral e, consequentemente, a melhoria do estado de saúde dos trabalhadores. 


\section{REFERÊNCIAS}

1. Lipp M. Estresse emocional: a contribuição de estressores internos e externos. Rev Psiq Clínica 2001; 28(6):347-349.

2. Karasek RA, Theorell T. Healthy work. Stress, productivity and the reconstruction of working life. New York: Basic Books; 1990.

3. Araújo TM, Graça CC, Araújo E. Estresse ocupacional e saúde: contribuição do modelo demanda-controle. Ciênc Saúde Coletiva 2003;8(4):991-1003.

4. Alves MGM. Pressão no trabalho: estresse no trabalho e hipertensão arterial em mulheres no estado pró-saúde. Rio de Janeiro. Tese[Doutorado em Saúde Pública] - Escola Nacional de Saúde Pública; 2004.

5. Fischer FM, Oliveira DC, Nagai R, Teixeira LR, Lombardi júnior M, Cooper SP . Job control, job demands, social support at work and health among adolescent workers. Rev Saúde Pública 2005;39(2): 245-253.

6. Reis EJFB, Carvalho FM, Araújo TM, Porto LA, Silvany Neto AM. Trabalho e distúrbios psíquicos em professores da rede municipal de Vitória da Conquista, Bahia, Brasil. Cad Saúde Pública 2005; 21(5):1480-1490.

7. Melchior M, Cspi A, milne BJ, Danese A, Poulton R, Moffitt TE. Work stress precipitates depression and anxiety in young, working women and men. Psychol Med 2007;37(8):1119-1129.

8. Rusli BN, Edimansyah BA, Naing L. Working conditions, self-perceived stress, anxiety, depression and quality of life: A structural equation modeling approach. BMC Public Health 2008;8(48):12.

9. Feng CK, Chen ML, Mao F. Prevalence of and risk factors for different measures of low back pain among female nursing aides in Taiwanese nursing homes. BMC Musculoskelet Disord [periódico na internet] 2007 [acesso em 10 jul 2008];8:52. Disponível em: http://www.ncbi.nlm. nih.gov/pubmed/17593305

10. Magnago TSBS. Aspectos psicossociais do trabalho e distúrbios musculoesqueléticos em trabalhadores de enfermagem. Rio de Janeiro.Tese [Doutorado em Enfermagem] - Universidade Federal do Rio de Janeiro; 2008.

11. Reis EJFB, Araújo TM, Carvalho FM, Barbalho L, Silva MO. Docência e exaustão emocional. Educação e Sociedade 2006;27(94):229-253.

12. Higashiguchi KK, Nakagawa $H$, Morikawa $Y$, Ishizaki $M$, Miura K, Naruse $Y$, et al. The association between job demand, control and depression in workplaces in Japan. J Occup Health 2002;44(6):427-428.

13. Schmidt DRC. Qualidade de Vida no Trabalho e sua associação com o estresse ocupacional, a saúde física e mental e o senso de coerência entre profissionais de enfermagem do Bloco Cirúrgico. Ribeirão Preto. Tese [Doutorado em Enfermagem] - Escola de Enfermagem de Ribeirão Preto da USP; 2009.

14. Alves MGM, Chor D, Faerstein E, Lopes CS, Werneck GL. Versão resumida da "Job Stress Scale": adaptação para o português. Rev Saúde Pública 2004;38(2):164-171.
15. Mendes KDS, Silveira RCCP, Galvão MC. Revisão integrativa: método de pesquisa para a incorporação de evidências na saúde e na enfermagem. Texto \& contexto Enferm 2008; 17(4):758-64.

16. Ganong LH. Integrative Reviews of nursing research. Res Nurs Health 1987;10(1):1-11.

17. Araújo TM, Aquino E, Menezes G, Santos CO, Aguiar L. Aspectos psicossociais do trabalho e distúrbios psíquicos entre trabalhadores de enfermagem. Rev Saúde Pública 2003;37(4):424-433.

18. Amaral TR. Dimensões psicossociais do trabalho da enfermagem e os distúrbios psíquicos menores em unidades críticas. Florianópolis. Dissertação [Mestrado em Enfermagem] - Universidade Federal de Santa Catarina; 2006.

19. Pinho PS, Araújo TM. Trabalho de Enfermagem em uma unidade hospitalar e transtornos mentais. Rev Enferm UERJ 2007;15(3):329-336.

20. Silva JLL. Estresse e transtornos mentais em trabalhadores de enfermagem. Rio de Janeiro. Dissertação [Mestrado em Enfermagem] - Universidade Federal do Estado do Rio de Janeiro; 2007.

21. Simão AAG, Siqueira Júnior AC, Ferreira CA, Mastini DP. Estresse em uma Unidade de Terapia Intensiva. Rev Nursing 2008;11(125):468-471.

22. Silva LG, Yamada KN. Estresse ocupacional em trabalhadores de uma unidade de internação de um hospital escola. Ciênc Cuid Saúde 2008;7(1):98-105.

23. Dalarosa MG, Lautert L. Accidents with biological materials among nurses in a training hospital: case-control study. Rev Gaúcha Enferm 2009;30(1):19-26.

24. Kirchhof ALC, Magnago TSBS, Camponogara S, Griep RH, Tavares JP, Prestes FC, et al. Condições de trabalho e características sócio-demográficas relacionadas à presença de distúrbios psíquicos menores em trabalhadores de enfermagem. Texto \& Contexto Enferm 2009;18(2):215-223.

25. Schmidt DRC, Dantas RAS, Marziale MHP, Laus AM. Estresse ocupacional entre profissionais de enfermagem do Bloco Cirúrgico. Texto \& Contexto Enferm 2009;18(2):330-337.

26. Fogaça MC, Carvalho WB, Nogueira PCK, Martins LAN. Estresse ocupacional e suas repercussões na qualidade de vida de médicos e enfermeiros intensivistas e neonatais. Rev Bras Ter Intensiva 2009; 21(3):299-305.

27. Souza MCB, Santos TCMM, Pinheiro MF, Freitas NA, Mendes RG, Pires TPA. Estresse ocupacional de equipe de enfermagem do Centro Cirúrgico. Rev Enferm UFPE on line 2009;3(3):86-96.

28. Negeliskii C. O estresse laboral e a capacidade para o trabalho de enfermeiros no Grupo Hospitalar Conceição. Porto Alegre. Dissertação[Mestrado em Enfermagem] -Universidade Federal do Rio Grande do Sul; 2010.

29. Griep RH, Rotenberg L, Landsberg P, Silva PRV. Uso combinado de modelos de estresse no trabalho e a saúde auto-referida na enfermagem. Rev Saúde Pública 
2011;45(1):145-152.

30. Silva JLL, Nóbrega ACR, Brito FCF et al. Tensão no trabaIho e a prevalência de transtornos mentais comuns entre trabalhadores de enfermagem. Rev Enferm UFPE on line 2011;5(1):1-9.

31. Magnago TSBS, Lisboa MTL, Griep RH, Zeitoune RCG, Tavares JP. Condições de trabalho de profissionais da enfermagem: avaliação baseada no modelo Demanda-Controle. Acta Paul Enferm 2010; 23(6):811-817.

32. Peter R, Siegrist J, Hallqvist J, Reuterwall C, Theorell T, Sheep Study Group. Psychosocial work environment and myocardial infarction: improving risk estimation by combining two complementary job stress model in the SHEEP study. J Epidemiol Community Health 2002;56(4): 294-300.

33. Rosvall $M$, Östergren PO, Hedblad B, Isacsson SO, Janzon L, Berglund G. Work-related psychosocial factors and carotid atherosclerosis. Intern J Epidemiology 2002;31(6):1169-1178.

34. Wamala SP, Mittleman MA, Horsten M, Schenck-Gustafsson K, Orth-Gómer K. Job stress and the occupational gradient in coronary heart disease risk in women: the stockholm female coronary health study. Soc Sci Med 2000;51(4):481-489. 